\title{
Local Defect Resonance (LDR): A Route to Highly Efficient Thermosonic and Nonlinear Ultrasonic NDT
}

\author{
Igor Solodov \\ University of Stuttgart, Institute of Polymer Technology, \\ Department of Non-Destructive Testing (IKT-ZfP), 70569 Stuttgart, Germany
}

\begin{abstract}
The concept of LDR is based on the fact that inclusion of a defect leads to a local drop of rigidity for a certain mass of the material that should manifest in a particular characteristic frequency of the defect. A frequency match between the driving ultrasonic wave and this characteristic frequency provides an efficient energy pumping from the wave directly into the defect. For simulated and realistic defects in various materials the LDR-induced local resonance increase in the vibration amplitude averages up to $\sim(20-40 \mathrm{~dB})$. Due to a strong resonance amplification of the local vibrations, the LDR-driven defects manifest a profound nonlinearity even at moderate ultrasonic excitation level. The nonlinearity combined with resonance results in efficient generation of the higher harmonics and is also used as a filter/amplifier in the frequency mixing mode of nonlinear NDT. The LDR high-Q thermal response enables to realize a frequency-selective imaging with an opportunity to distinguish between different defects by changing the driving frequency. The LDR-thermosonics requires much lower acoustic power to activate defects that makes it possible to avoid high-power ultrasonic instrumentation and proceed to a noncontact ultrasonic thermography by using air-coupled ultrasonic excitation.
\end{abstract}

Keywords: Local Defect Resonance, Ultrasonic Thermography, Nonlinear Nondestructive Testing

PACS: 43.25.-x; 87.63.Hg

\section{INTRODUCTION}

Ultrasonic nonlinearity and thermosonics (also called vibro-thermography or ultrasonic thermography) are promising approaches to NDT of materials and industrial components based, correspondingly, on nonlinear and thermal responses of defects exposed to ultrasonic excitation. They are both caused by relatively inefficient processes of clapping and/or frictional hysteretic rubbing at the defect interface and therefore depend critically on input ultrasonic power. As a result, both methodologies stand out from other conventional ultrasonic NDT counterparts for their specific instrumentation particularly adapted to high-power ultrasonics. The nonlinear techniques usually require a low-klirfactor generators and transducers combined with thorough filtering of higher harmonics barely possible for the high-voltage (hundreds volts) inputs. To provide a measurable temperature response, thermosonics traditionally employs high-power ultrasonic welding instrumentation, which includes $\mathrm{kW}$ power supply (at fixed frequencies 20 or $40 \mathrm{kHz}$ ) and piezo-stack converters combined with ultrasonic boosters and horns [1]. The test specimen is usually pressed against the horn that results in unstable ultrasonic response and highly non-reproducible measurements.

In both cases, an increase in efficiency was found by using a resonant excitation of the specimen providing that the defect is outside the nodal areas of the standing wave pattern [2,3]. This condition was shown to enhance the nonlinear defect response (higher harmonics, combination frequencies, etc.) and was applied in multiple experiments on nonlinear characterization of materials, e.g. [4]. In order to diminish the effect of nodal lines and to exclude "missing" the defect, the multi-mode analytical approach was developed [5]. It is based on selection of the specimen resonance modes with particularly high nonlinear responses for a given defect location.

A possible drawback of a single-frequency ultrasonic excitation concerned with the nodal lines was also realized in ultrasonic thermography. To increase the probability of detecting a crack it was suggested to excite the specimens at multiple resonance frequencies instead of using a single-frequency excitation [6]. An enhancement in crack detectability by producing a wide-band excitation ("acoustic chaos") was reported in [7].

In this paper, a consistent way to enhance nonlinear acoustic and thermal defect responses is suggested by using selective ultrasonic activation of defects based on the concept of local defect resonance [8]. The LDR provides a selective excitation of a defect to result in a high local vibration amplitude and enhancement of both nonlinear and thermal defect responses readily measurable even for a few $\mathrm{mW}$ acoustic input. 


\section{EXPERIMENTAL EVIDENCE FOR LDR}

A direct way to experimentally reveal LDR is to measure an individual contribution of each point of the specimen in its overall frequency response in a wide frequency range. For this purpose, an ultrasonic excitation by a wide-band piezoelectric transducer is combined with a laser vibrometer scan of the specimen surface. It enables to probe and indicate all possible resonances in every point of the specimen. The origin of each maximum is then verified by imaging the wave pattern in the specimen at the corresponding frequency. Fig. 1 shows an example of the LDR frequency response (a) and the vibration pattern (b) measured for a simulated defect (flat-bottomed hole $(\mathrm{FBH})$ ) in a PMMA plate. A strong enhancement (about $20 \mathrm{~dB}$ ) of the vibration amplitude with a high Q-factor $(Q \approx 70)$ observed locally in the defect area is identified as a fundamental defect resonance (Fig. 1). Besides the fundamental LDR, zoom-in scan of the vibration field inside the defect area in a wider frequency range reveals the higher-order LDR with multiple nodal lines in the vibration patterns. Such a methodology was successfully applied by us to a search for LDR in a variety of materials [8]. The two examples presented in Figs. 2, a, b illustrate a clear evidence of LDR in kHz-frequency range for cracks and impacts in composite and constructional components. Similar LDR with local resonance "amplification" of the vibration amplitude as high as $\sim(20-40 \mathrm{~dB})$ were generally measured for other types of realistic defects.
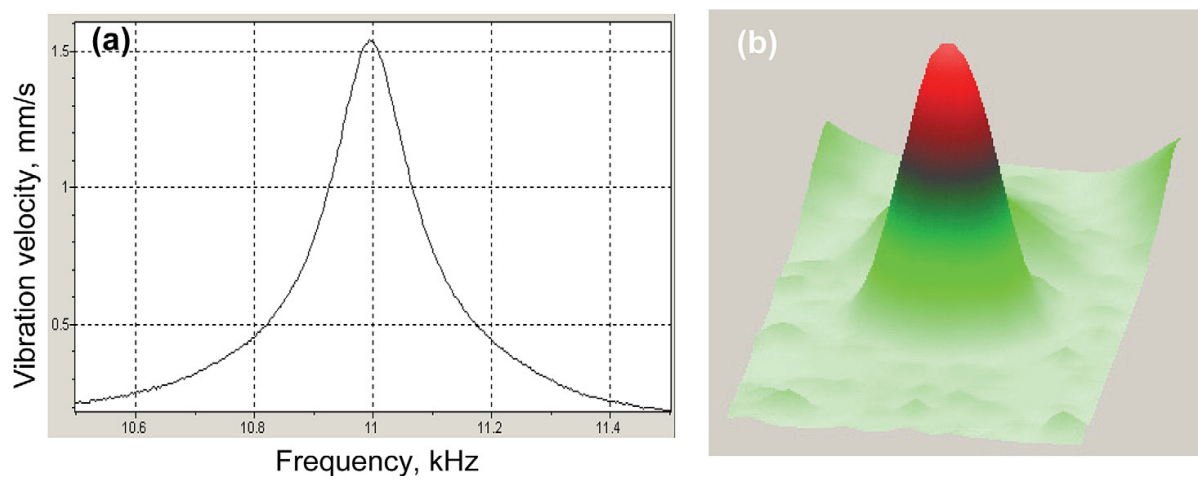

FIGURE 1. Frequency response (a) and vibration pattern at $11 \mathrm{kHz}$ (b) for LDR in a circular FBH (10 mm radius and thickness $0.8 \mathrm{~mm})$ in PMMA plate $\left(200 \times 40 \times 4 \mathrm{~mm}^{3}\right)$.
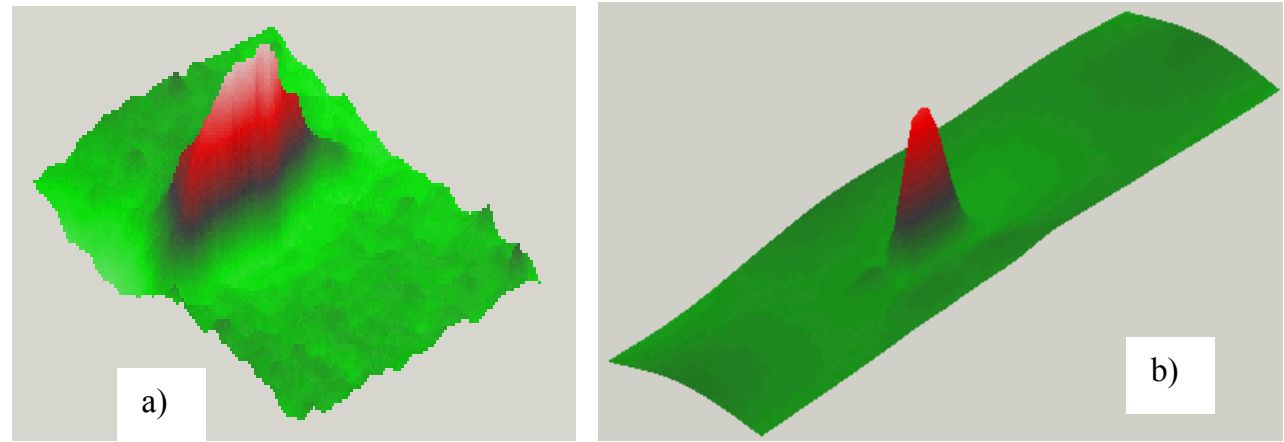

FIGURE 2. LDR vibration patterns for a $50 \mu \mathrm{m}$-wide and $10 \mathrm{~cm}$-long crack in GFR-concrete specimen (frequency 4.19 $\mathrm{kHz},(\mathrm{a}))$ and for an impact induced loss of fibers (area $25 \times 2 \mathrm{~mm}^{2}$ ) in a CFRP plate (frequency $3.66 \mathrm{kHz}$, (b)).

\section{LDR NONLINEARITY FOR DEFECT IMAGING AND NDT}

Strong vibrations provided by LDR locally in the defect area should cause nonlinear effects even for simulated defects (FBH) driven by a relatively inefficient mechanism of material ("classical") nonlinearity. Much higher nonlinearity is manifested by a variety of realistic cracked defects (delaminations, impacts, cracks, etc.) due to some different mechanisms (contact acoustic nonlinearity (CAN), hysteretic nonlinearity, etc.). By combining the resonance conditions provided by the LDR with highly-efficient elastic nonlinearity (e.g. CAN) a substantial improvement in detecting and imaging of realistic defects by means of nonlinear NDT can be expected. 
A strong increase in defect nonlinearity due to LDR is illustrated in Fig. 3 for a crack in a unidirectional (UD-) carbon fibre-reinforced (CFRP) rod. The zoom-in frequency response of the defect obtained for a wide-band excitation (Fig. 3, (a)) reveals the LDR-frequency $f_{0} \approx 19500 \mathrm{~Hz}$. As the driving frequency matches the LDRfrequency, a drastic enhancement $(\sim 20-40 \mathrm{~dB})$ of the higher-order harmonic $(\mathrm{HH})$ amplitudes generated locally in the defect area is observed (Fig. 3, (b)).

Under the LDR condition, the higher harmonics are generated efficiently and highly localized in the defect area that provides a background for the high-contrast defect-selective imaging. The benefit of the higher harmonic LDR imaging is illustrated in Fig. 4. A substantial improvement of the image quality $\left(\sim 10 \times 20 \mathrm{~mm}^{2}\right.$ delamination in 1 mm-glass fiber-reinforced (GFRP) plate) is clearly seen by comparing the fundamental ((a), signal-to-noise ratio $\sim 12 \mathrm{~dB})$ ) and the second harmonic ((b), signal-to-noise-ratio $\sim 24 \mathrm{~dB})$ images.
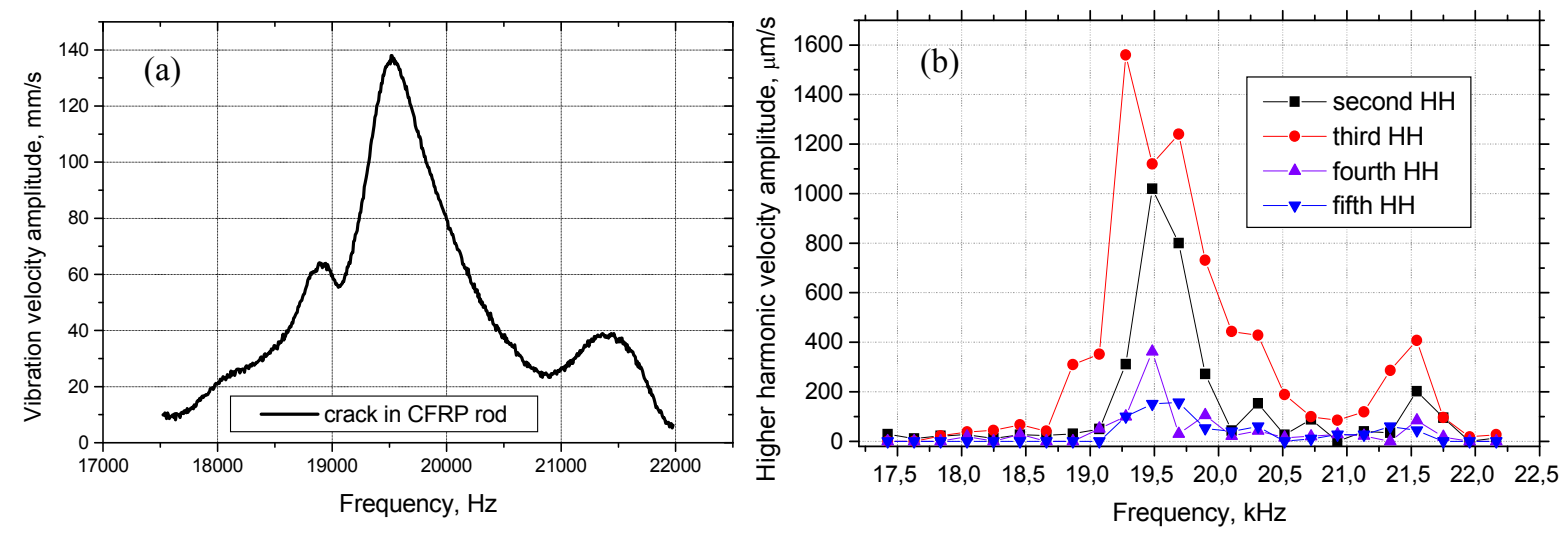

FIGURE 3. Linear (a) and higher harmonic (b) LDR frequency response of a crack in a UD-CFRP rod.

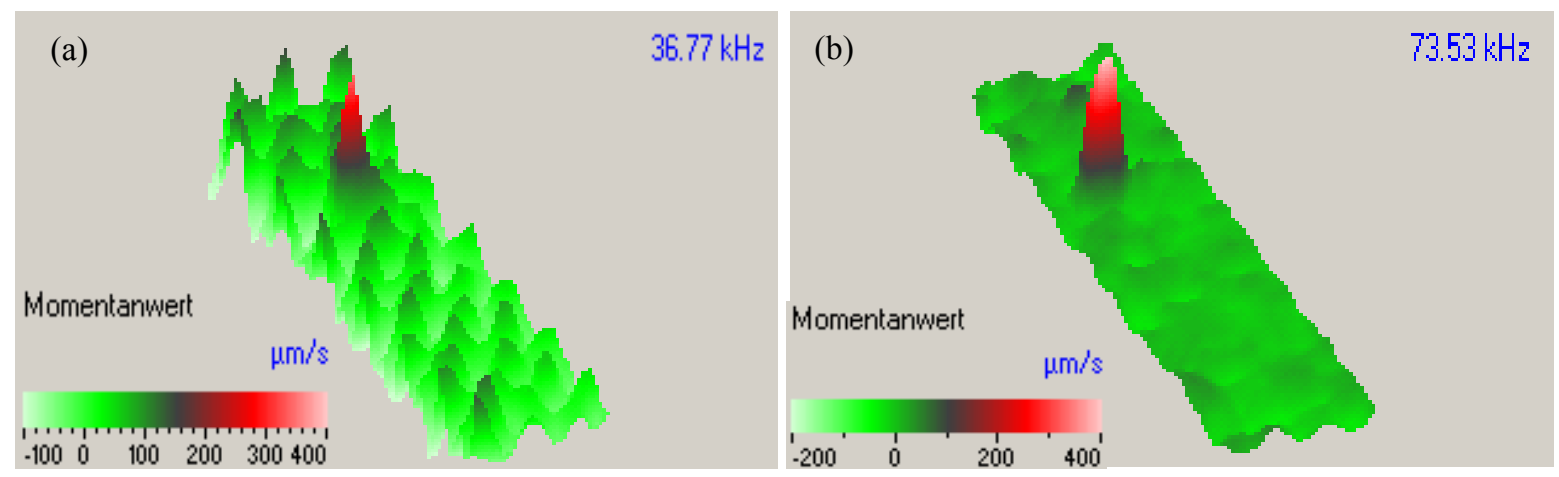

FIGURE 4. Improvement of imaging quality $\left(10 \times 20 \mathrm{~mm}^{2}\right.$ GFRP delamination) by using higher harmonic LDR: (a) image at fundamental LDR (36.77 kHz); (b) second harmonic image $(73.53 \mathrm{kHz})$.

A high quality factor of LDR can also be used as a "linear" filter/amplifier in the frequency mixing nonlinear NDT. This method is based on the nonlinear interaction of ultrasonic waves of different frequencies $\left(f_{1}, f_{2}\right)$ that results in a combination frequency output: $f_{ \pm}=f_{1} \pm f_{2}$. For nonlinear experiments in "classical" materials (like, PMMA), the efficiency of interaction is highly critical to the geometry of the wave propagation and is generally rather low: the amplitude ratio $U_{ \pm} / U_{1,2}$ is normally below $\left(10^{-3}-10^{-2}\right)$. A high-Q-LDR can be used to enhance the output signal by a combination frequency (or any of the interacting frequencies) match to the LDR frequency response. This approach will be applicable to any geometry of the wave interaction since the LDR response is weakly sensitive to the defect position in the wavefield.

In the experiment, the LDR induced "amplification" of the nonlinear interaction was studied for the FBH in PMMA plate $\left(f_{0} \approx 11000 \mathrm{~Hz}\right.$ ) whose frequency response is shown in Fig. 1 . The two interacting flexural waves were excited in continuous wave mode by the piezo-transducers attached to the opposite edges of the plate. One of 
the frequencies was fixed at $f_{1}=25 \mathrm{kHz}$, while $f_{2}$ was varied in the range of $(13500-14400 \mathrm{~Hz})$ to provide the difference frequency $f_{-}=f_{1}-f_{2}$ to sweep the LDR bandwidth of the FBH. The vibration velocity amplitudes ( $V$ ) at $f_{1}, f_{2}$ and $f_{-}$were monitored in the centre of FBH with a laser scanning vibrometer (vibration velocity mode).

Fig. 5 shows the normalized velocity amplitude at difference frequency as a function of $f_{-}$measured by changing $f_{2}$ in the frequency range indicated above. The effect of LDR is clearly seen by comparing the data with those in Fig. 1. To quantify the amplification effect we estimate the interaction efficiency factor $\eta=V \_/ V_{1} V_{2}[\mathrm{~s} / \mathrm{m}]$ inside and outside the LDR bandwidth. The values obtained, correspondingly: $\eta(11050) \cong 100$ and $\eta(10600) \cong 1.6$ illustrate a strong increase in efficiency induced by LDR. The resonance amplification of nonlinear vibrations also provides unusually high amplitude of mixed frequency signal: at the optimal frequency match $\left(f_{-}=f_{0}\right)$, the amplitude ratio $V \_/ V_{2} \cong 44 \%$ and $V \_/ V_{1} \cong 93 \%$.

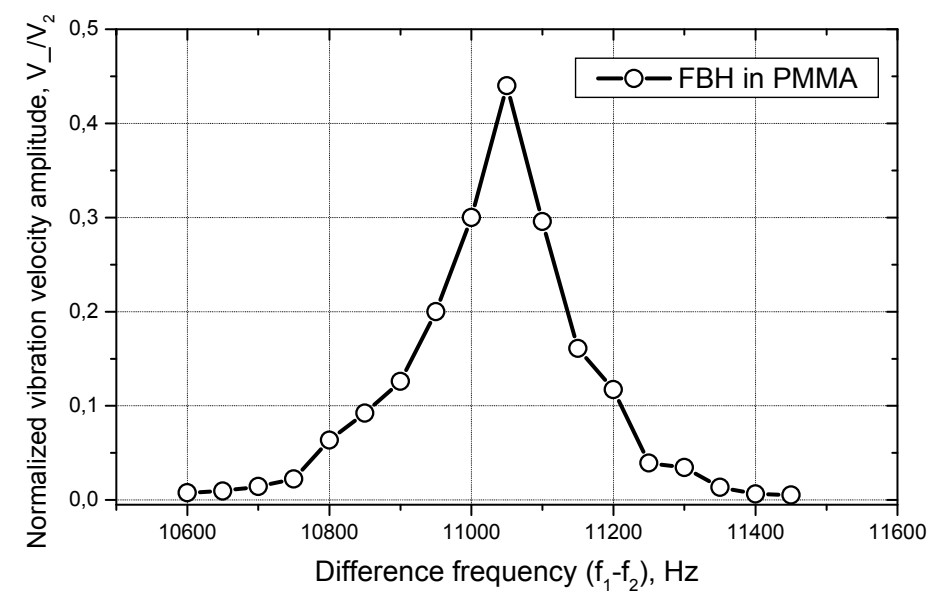

FIGURE 5. LDR induced amplification of the difference frequency vibration measured for an FBH in PMMA specimen.

\section{LDR THERMOSONICS}

In ultrasonic thermography, the defect thermal response is caused by a local dissipation of mechanical energy, which is converted into heat. In the frame of viscoelastic model, this process is described by introducing the internal friction force proportional to velocity of vibration $\sigma \sim E^{\prime \prime} \dot{\varepsilon}$, so that energy loss per unit time is: $\Delta W / \Delta t=\omega^{2} \varepsilon_{0}^{2} E ` / 2$. The dissipated power is, therefore, proportional to the square of both the frequency $(\omega)$ and the amplitude $\left(\varepsilon_{0}\right)$ of vibration. Hence, the use of LDR, which strongly intensifies local vibrations, is beneficial for enhancing the efficiency of ultrasonic thermography.

In the experiments, the effect of LDR on thermal response of defects was studied for circular FBH of different sizes in PMMA plates as well as for realistic defects in composites. Unlike traditional ultrasonic thermography experiments, which usually apply hand-pressed horn-type transducers for exciting high-power vibrations, we used conventional disk-like piezo-ceramic transducers attached (glued) to the specimen surface. The input voltage up to $90 \mathrm{~V}$ amplitude from HP 33120A function generator (bandwidth up to $15 \mathrm{MHz}$ ) via a high voltage amplifier HVA $3 / 450$ was applied to the transducers to excite the flexural waves in the frequency band up to $100 \mathrm{kHz}$. The standing wave amplitudes were monitored with scanning laser vibrometer to evaluate a total acoustic power injected in the specimens; it was found to be in the sub-watt range even for maximum input voltage. The thermal response of the defects was visualized and measured with an IR-camera (IRCAM Equus $327 \mathrm{~K}$, sensitivity $\approx 20 \mathrm{mK}$ ).

Fig. 6, (a) shows an example of thermosonic image for a circular FBH (radius $1 \mathrm{~cm}$ ) excited at LDR frequency $(11 \mathrm{kHz})$; the dynamics of the FBH thermal response is illustrated in Fig. 6, (b). A quadratic dependence on the input amplitude agrees fully with theoretical expectations. The data also reveal a high efficiency of the vibro-thermal conversion: at $80 \mathrm{~V}$ input $(0.2 \mathrm{~W}$ acoustic power) and $15 \mathrm{~s}$-ultrasonic exposure, the temperature rise in the $\mathrm{FBH}$ 
amounts to $\approx 3 \mathrm{~K}$. According to Fig. 6 , (b), the thermal response stays substantially beyond the camera sensitivity down to $15-20 \mathrm{~V}$ input ( $\sim 10 \mathrm{~mW}$ acoustic power).

A crucial contribution of the LDR to the heating effect is clarified by measurements of the temperature rise as a function of driving frequency (Fig. 7, experimental points). Even 2-3\% detuning from an exact LDR frequency drops the temperature down to basically non-measurable values of 10-20 mK and reduces the conversion efficiency by about two orders of magnitude. Such a high-Q thermal response is a consequence of the nonlinearity involved in the acousto-thermal conversion. This fact is illustrated in Fig. 7 by a close fit between the LDR frequency response of the FBH (shown in Fig. 1, (a)) squared and its thermal response.
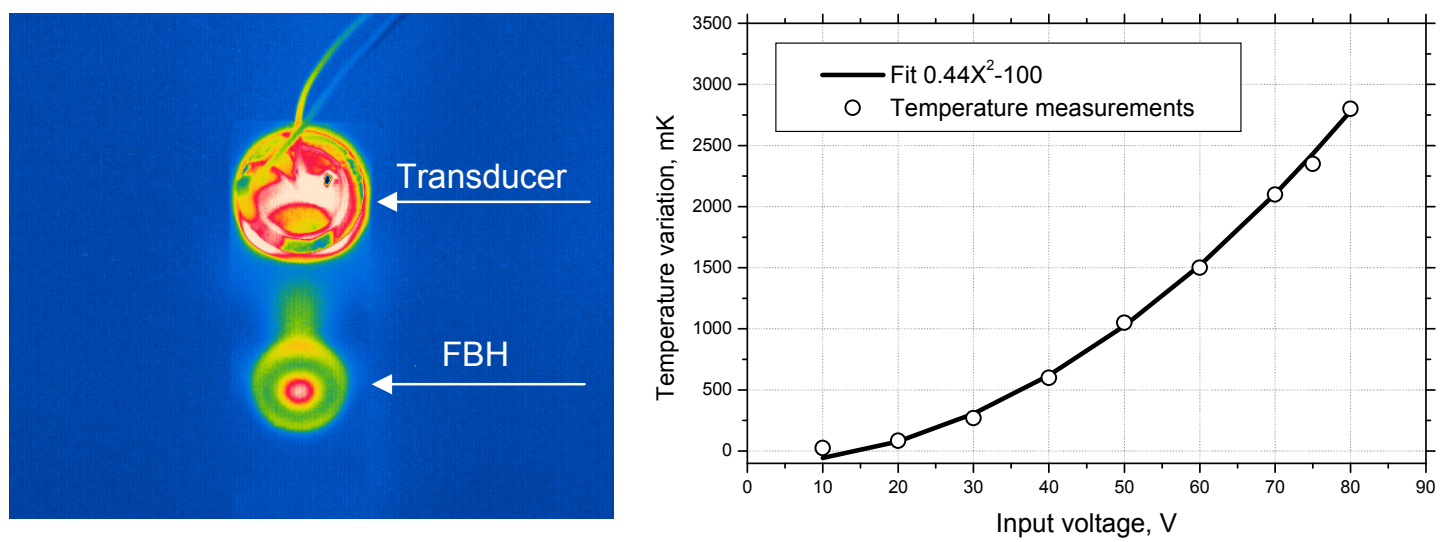

FIGURE 6. (a) - thermosonic image of a circular FBH (radius $1 \mathrm{~cm}$ ) in PMMA plate at LDR frequency $(11 \mathrm{kHz})$. Insonation time $15 \mathrm{~s}$; input voltage of the transducer $80 \mathrm{~V}$; (b)- temperature variation of a FBH at LDR as a function of input voltage.

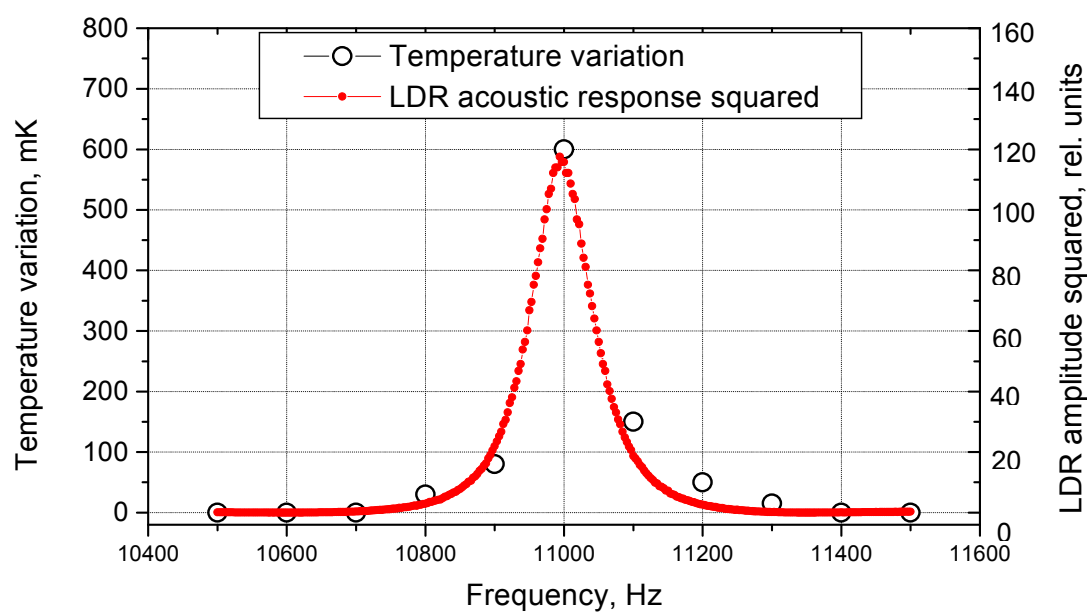

FIGURE 7. Comparison between FBH vibro-thermography response and LDR acoustic frequency response (shown in Fig. 1) squared.

A high thermal quality factor of the LDR enables to realize an advanced defect-selective imaging that combines a general selective defect imaging intrinsic to ultrasonic thermography with an opportunity to distinguish between different defects by changing the driving frequency. To demonstrate a feasibility of this mode we used a matrix of 16 circular FBH $(r=5 \mathrm{~mm})$ of different thicknesses (and corresponding LDR frequencies) in a disk-like PMMA specimen. As the excitation frequency changed, both the acoustic (measured with the scanning vibrometer) and the thermal responses of the defects were monitored. The images in Fig. 8 confirm that the defect positions synchronously "switch over" as soon as the driving frequency hits the LDR frequency of a particular FBH.

To proceed to realistic defects we studied the effect of LDR on the thermal response for an in-plane oval delamination in a GFRP plate. The results are presented in Figs. 9, 10, where the thermal responses to a 2s-pulse acoustic excitation of the delamination are visualized (Fig. 9) and measured (Fig. 10) for the same input amplitudes $(50 \mathrm{~V})$ but different frequencies. The thermographic image of the defect taken in the vicinity of the LDR $\sim 20.9 \mathrm{kHz}$ (Fig. 9 (b)) demonstrates that the heating is mainly produced in the core part of the delamination where maximum 
vibration amplitude is observed (Fig. 9 (a)). The temperature variation in this area (for insonation time 2 s) (Fig. 10) confirms the resonance character of the effect: At the LDR central frequency, the temperature rise $\left(0.85{ }^{\circ} \mathrm{C}\right)$ is almost an order of magnitude higher than that outside the LDR bandwidth. For larger deviation of the driving frequency from LDR (including the specimen natural frequencies), the thermal response of the defect was barely measurable with the IR-camera (sensitivity $\approx 20 \mathrm{mK}$ ).

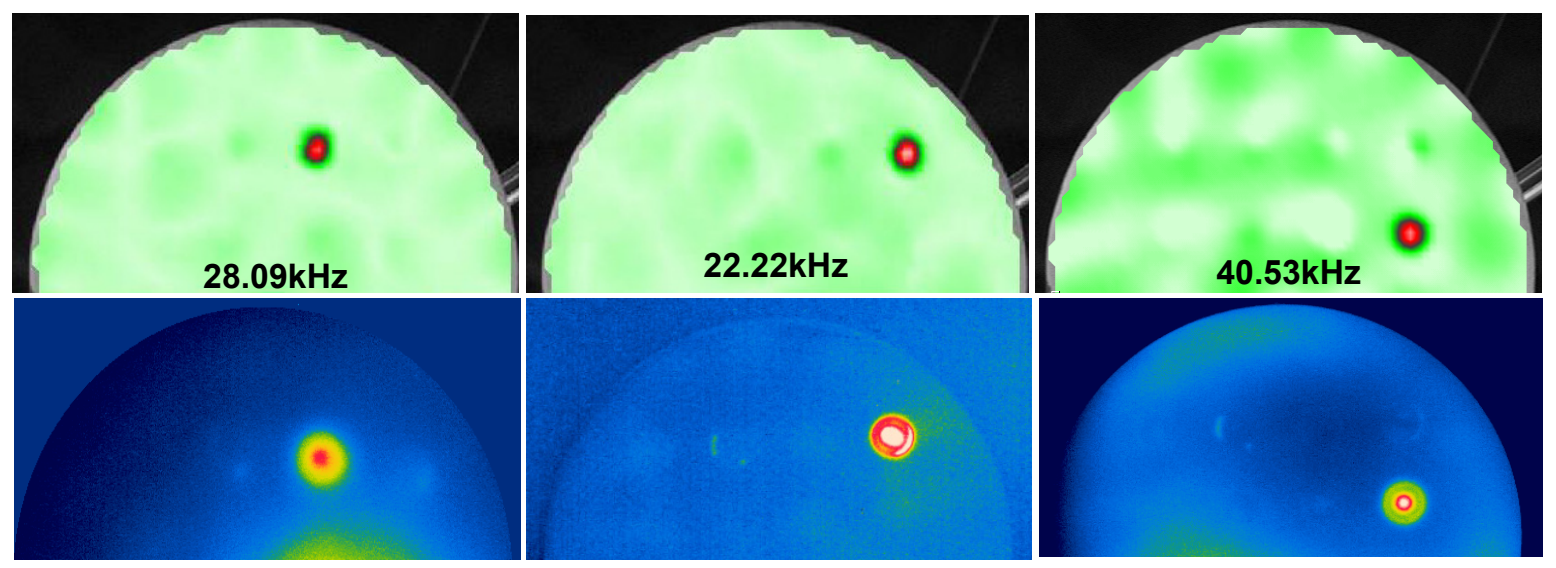

FIGURE 8. Laser vibrometry (top) and thermosonic (bottom) images of FBH driven at different LDR frequencies in a PMMA disk specimen.
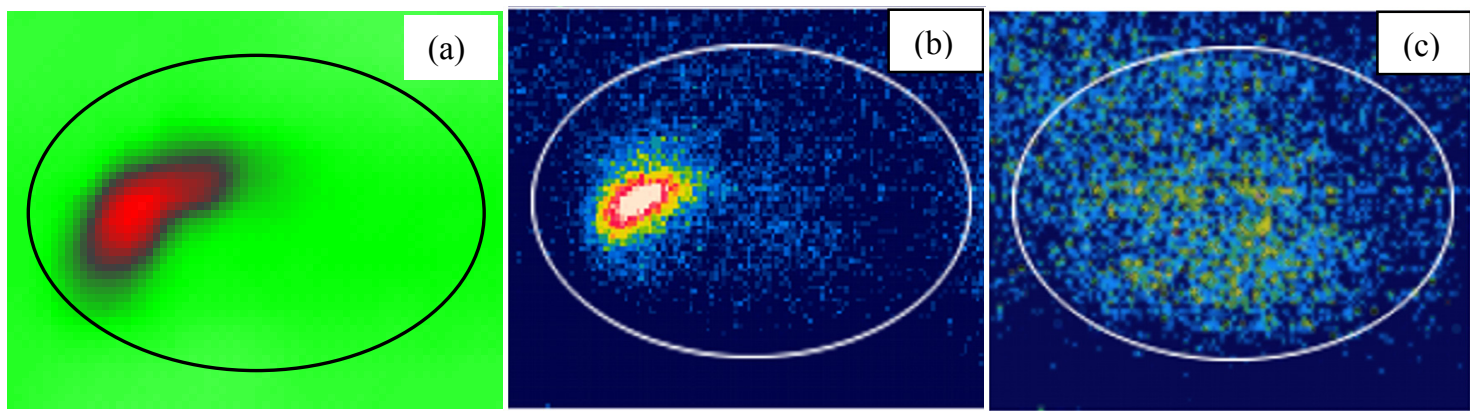

FIGURE 9. Resonance imaging of an oval delamination in GFRP specimen: $20.9 \mathrm{kHz}-\mathrm{LDR}$ vibrometer (a) and IR-(b) images; (c) an IR-image at the specimen natural frequency $6.8 \mathrm{kHz}$.

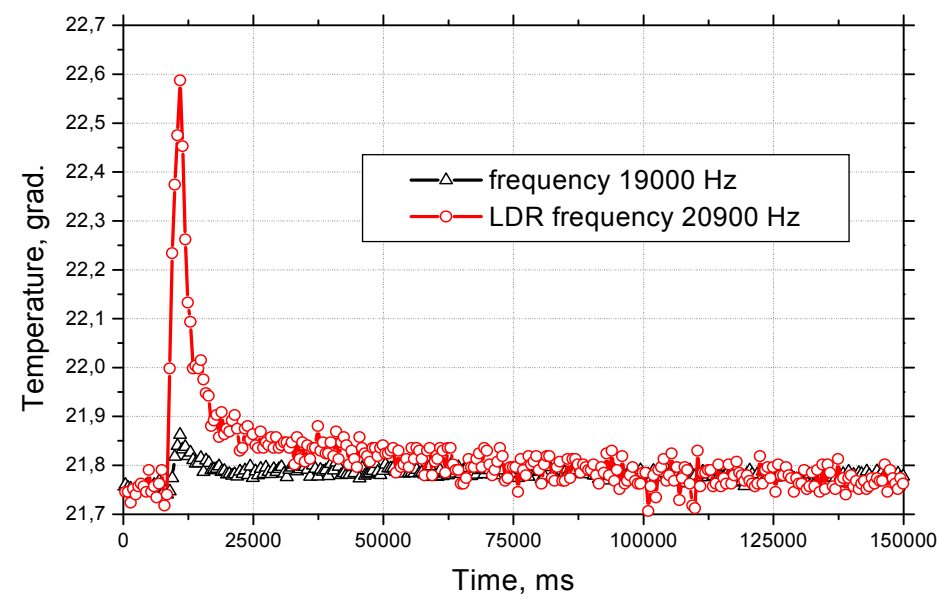

FIGURE 10. Thermal 2s-pulse responses of delamination at LDR-frequency $(20900 \mathrm{~Hz})$ and outside the LDR $(19000 \mathrm{~Hz})$. 
The results shown above imply that a strong increase in thermal output signal at LDR frequency enhances a signal-to-noise ratio (SNR) of the vibro-thermography imaging. On the other hand, an increase of the SNR is also known to occur in the lock-in ultrasonic thermography mode [9] primarily due to diminishing the noise level. By introducing the benefit of LDR in the lock-in approach a resonance vibro-thermography mode operating at unusually low excitation levels can be projected.

To this end, the amplitude of ultrasonic excitation at the LDR frequency $(11 \mathrm{kHz})$ was modulated sinusoidally by the lock-in frequency (between $0.01 \mathrm{~Hz}$ and $1 \mathrm{~Hz}$ ). A temperature image sequence of the surface was recorded with the IR-camera and a discrete Fourier transformation at the lock-in-frequency was applied to compress this image sequence into a pair of amplitude and phase images. An enhancement in sensitivity and the SNR of the LDR lock-in imaging are readily seen from Fig. 11, where the phase lock-in (a) and conventional temperature (b) vibrothermography images of the FBH are shown. Only $\approx 3 \mathrm{~mW}$ of the input acoustic power used in this experiment appear to suffice for reliable LDR lock-in imaging (Fig. 11, (a)) but be too low for a conventional temperature image to develop noticeably even at the LDR condition.
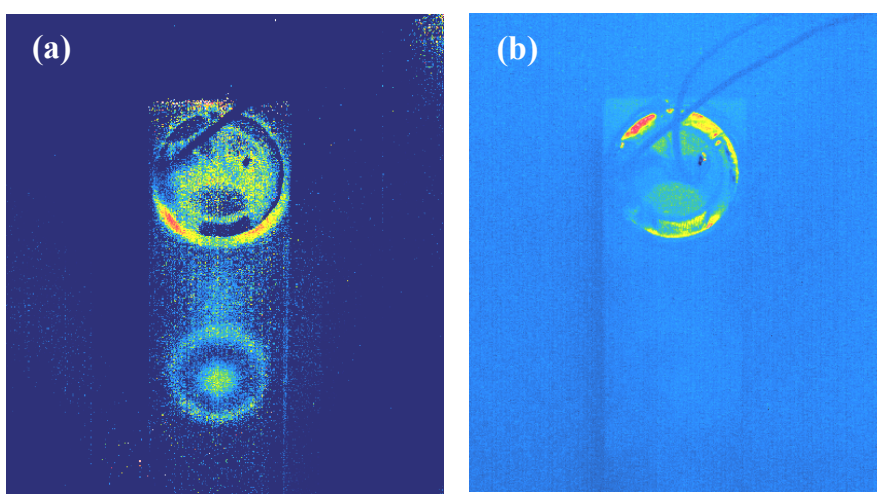

FIGURE 11. LDR lock-in phase (modulation $0.02 \mathrm{~Hz}$, (a)) and conventional temperature (60s insonation, (b)) images of a circular FBH in PMMA plate at LDR frequency $11 \mathrm{kHz}$.

Such an efficient ultrasonic activation enables to proceed with a noncontact thermosonics mode by using an aircoupled (ACU) excitation of defects. For this purpose, we used the Ultran ACU transducers whose fundamental frequencies $(\sim 50$ and $\sim 70 \mathrm{kHz})$ match the LDR frequencies of defects. The transducers were placed a few $\mathrm{cm}$ away from the defect area, while the IR-image was observed from the opposite side of the plate specimen (ACU-IR noncontact "through transmission" mode). The AC-radiometer methodology was employed to measure the ACU power radiated.

A temperature ACU thermosonic image of a $\mathrm{FBH}(3 \mathrm{~mm}$ radius, $1 \mathrm{~mm}$ thickness, $50 \mathrm{kHz} \mathrm{LDR}$ ) in a PMMA plate $\left(170 \times 50 \times 10 \mathrm{~mm}^{3}\right)$ is shown in Fig. 12 (a). For input ACU power of $\sim 50 \mathrm{~mW}$ and $30 \mathrm{~s}$ insonation pulse, the temperature rise amounts to $\sim 0.6 \mathrm{~K}$ (Fig. 12 (b)) that is far beyond the sensitivities of modern IR-cameras.
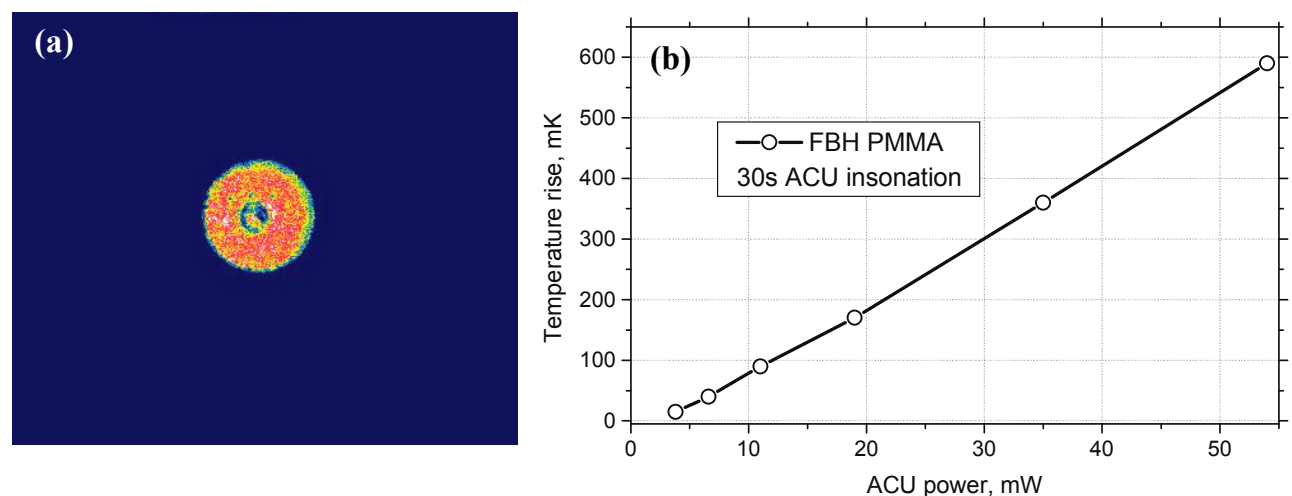

FIGURE 12. Temperature ACU thermosonic image of FBH ( $3 \mathrm{~mm}$ radius, $1 \mathrm{~mm}$ thickness) in PMMA plate (50 kHz LDR excitation, $54 \mathrm{~mW}$ ACU input power (a)); LDR induced temperature rise as a function of ACU power (b). 
An example of noncontact thermosonic imaging of realistic defects is illustrated in Fig. 13 for impact damage (LDR frequency $\sim 69.6 \mathrm{kHz}$ ) induced on the rear side in $1.1 \mathrm{~mm}$-thick multi-ply CFRP plate (Fig. 13 (a)). The ACU thermosonic phase image (Fig. 13 (c)) of the circular shape damage (radius $\sim 12 \mathrm{~mm}$ ) is taken in the lock-in mode for the input ACU power of $\sim 7 \mathrm{~mW}$. The similarity between the laser vibrometry (b) and ACU LDR thermosonic (c) images confirms the practical relevance of this noncontact thermography mode.
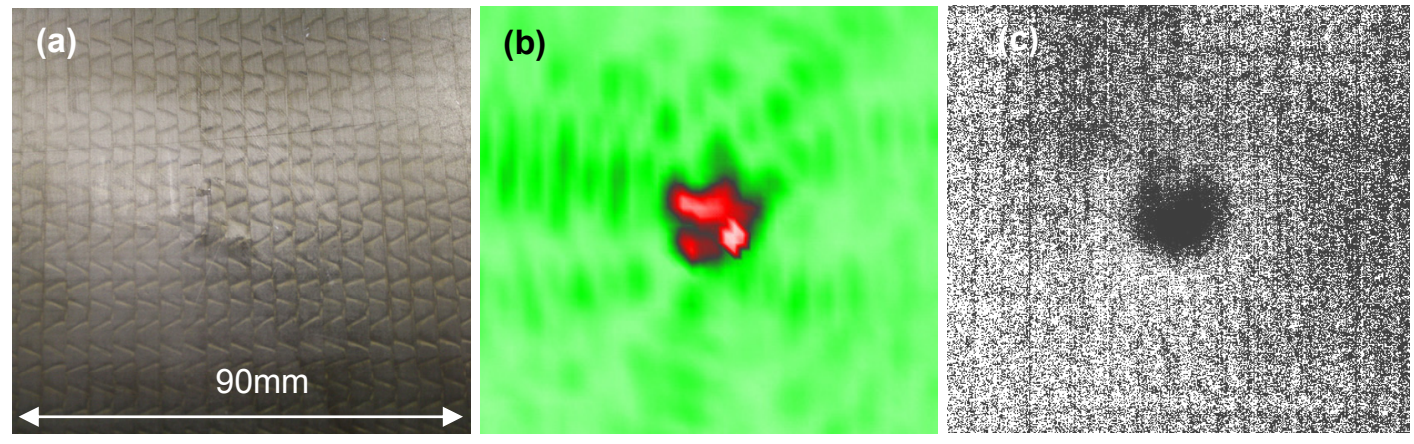

FIGURE 13. Imaging of impact-induced damage in multi-ply CFRP plate (photo, (a)); laser vibrometry image (b) and ACU-thermosonic lock-in phase image (modulation $0.03 \mathrm{~Hz},(\mathrm{c})$ ) at LDR frequency $69.6 \mathrm{kHz}$.

\section{CONCLUSIONS}

The experimental evidence of LDR obtained for simulated and realistic defects in a variety of materials demonstrates a local resonance "amplification" of the vibration amplitude up to $\sim(20-40 \mathrm{~dB})$. Since LDR is an efficient resonant "amplifier" of the local vibrations, it manifests a profound nonlinearity even at moderate ultrasonic excitation level. Besides a strong higher harmonic response, LDR can also be used as a "linear" filter/amplifier in the frequency mixing nonlinear NDT. The high LDR acoustic and thermal quality factors provide an opportunity for frequency-selective NDT, which distinguishes between different defects by changing the driving frequency. Unlike traditional thermosonic experiments, the LDR-thermography requires substantially lower acoustic power to activate defects that makes it possible to use conventional ultrasonic NDT instrumentation. For input acoustic power well below $1 \mathrm{~W}$, the temperature rise about few $\mathrm{K}$ is normally measured for LDR thermosonics of defects in composite materials. The LDR methodology enables to enhance acousto-thermal conversion efficiency by more than an order of magnitude and proceed to noncontact thermosonic NDT by using ACU excitation.

\section{ACKNOWLEDGEMENT}

The author acknowledges support of this study by EU FP-7 in the framework of ALAMSA project.

\section{REFERENCES}

1. R.B. Mignogna, R.E. Green Jr., J.C. Duke, E.G. Henneke II, and K.L. Reifsnider, Ultrasonics, 19, 159-163 (1981).

2. R. Guyer and P. Johnson, Physics Today, 52, 30-36 (1999).

3. J. Renshaw, S. Holland, and R.B. Thompson, Appl. Phys. Letters, 93, 081914 (2008).

4. K.E.-A Van Den Abeele., P. Johnson, and A. Sutin, Res. Nondestr. Evaluation, 12, 17-30 (2000).

5. K. Van Den Abeele, J. Acoust. Soc. Am., 122 (1), 73-90 (2007).

6. C. Homma, M. Rothenfusser, J. Baumann., and R. Shannon, AIP Conference Proceedings 820, American Institute of Physics, Melville, NY, 2006, pp. 556-573.

7. Xiaoyan Han, Zhi Zheng, Wei Li, Sawar Islam, Janping Lu, V. Loggins, E. Yitamben., L.D. Favro, G. Newaz, and R.L. Thomas., J. Appl. Phys., 95, 3792-3797 (2004).

8. I. Solodov, J. Bai, S. Bekgulyan, and G. Busse, Appl. Phys. Letters, 99, 211911 (2011).

9. G. Busse, D. Wu, and W. Karpen, J. Appl. Phys., 71, 3962-3965 (1992). 